\title{
Combining directed evolution of pathway enzymes and dynamic pathway regulation using a quorum-sensing circuit to improve the production of 4-hydroxyphenylacetic acid in Escherichia coli
}

Yu-Ping Shen, Lai San Fong, Zhi-Bo Yan and Jian-Zhong Liu*

\begin{abstract}
Background: 4-Hydroxyphenylacetic acid (4HPAA) is an important building block for synthesizing drugs, agrochemicals, biochemicals, etc. 4HPAA is currently produced exclusively via petrochemical processes and the process is environmentally unfriendly and unsustainable. Microbial cell factory would be an attractive approach for 4HPAA production.

Results: In the present study, we established a microbial biosynthetic system for the de novo production of 4HPAA from glucose in Escherichia coli. First, we compared different biosynthetic pathways for the production of 4HPAA. The yeast Ehrlich pathway produced the highest level of 4HPAA among these pathways that were evaluated. To increase the pathway efficiency, the yeast Ehrlich pathway enzymes were directedly evolved via error-prone PCR. Two phenylpyruvate decarboxylase ARO10 and phenylacetaldehyde dehydrogenase FeaB variants that outperformed the wild-type enzymes were obtained. These mutations increased the in vitro and in vivo catalytic efficiency for converting 4-hydroxyphenylpyruvate to 4HPAA. A tunable intergenic region (TIGR) sequence was inserted into the two evolved genes to balance their expression. Regulation of TIGR for the evolved pathway enzymes further improved the production of 4HPAA, resulting in a 1.13-fold increase in titer compared with the fusion wild-type pathway. To prevent the toxicity of a heterologous pathway to the cell, an Esa quorum-sensing (QS) circuit with both activating and repressing functions was developed for inducer-free productions of metabolites. The Esa-P esaR $_{\text {activation } Q S}$ system was used to dynamically control the biosynthetic pathway of 4HPAA in E. coli, which achieved $17.39 \pm 0.26 \mathrm{~g} / \mathrm{L}$ with a molar yield of $23.2 \%$ without addition of external inducers, resulting in a $46.4 \%$ improvement of the titer compared to the statically controlled pathway.
\end{abstract}

Conclusion: We have constructed an E. coli for 4HPAA production with the highest titer to date. This study also demonstrates that the combination of directed evolution of pathway enzymes and dynamic pathway regulation using a QS circuit is a powerful strategy of metabolic engineering for the productions of metabolites.

Keywords: 4-Hydroxyphenylacetic acid, Directed evolution, Dynamic pathway regulation, Quorum-sensing system, Escherichia coli

\footnotetext{
*Correspondence: Issljz@mail.sysu.edu.cn

Institute of Synthetic Biology, Biomedical Center, Guangdong

Province Key Laboratory of Improved Variety Reproduction in Aquatic

Economic Animals, School of Life Sciences, Sun Yat-sen University,

Guangzhou 510275, China
}

(c) The Author(s) 2019. This article is distributed under the terms of the Creative Commons Attribution 4.0 International License (http://creativecommons.org/licenses/by/4.0/), which permits unrestricted use, distribution, and reproduction in any medium, provided you give appropriate credit to the original author(s) and the source, provide a link to the Creative Commons license, and indicate if changes were made. The Creative Commons Public Domain Dedication waiver (http://creativecommons.org/ publicdomain/zero/1.0/) applies to the data made available in this article, unless otherwise stated. 


\section{Background}

Phenolic acids are aromatic acids that contain a phenol ring and at least one organic carboxylic acid group. Phenolic acids play an important role in human health and have wide applications in food, cosmetic and pharmaceutical industries. 4-Hydroxyphenylacetic acid (4HPAA) has received much attention because of its numerous applications. 4HPAA is used in the synthesis of penicillin G, atenolol, benzoprofen, and agrochemicals, etc. [1, 2]. 4HPAA is an active component of Rhodiola rosea [2] and the Chinese herbs Aster tataricus (fan hun cao). Aster tataricus is widely used in China for the treatment of pneumonia, HBV, and carcinomas [3-5]. Furthermore, 4HPAA possesses anxiolytic [6], antiplatelet [7] and hepatoprotective [8] properties. In addition, 4HPAA was considered as a potential hypopigmenting agent [9] and an inhibitor of hypertonicity and hypoxia [10].

4HPAA can be obtained by chemical synthesis from different substrates such as anisol, $p$-cresol, phenol, benzyl phenyl ether, or hydroxymandelic acid [11, 12]. However, the chemical routes have some drawbacks, including a requirement of elevated temperatures and pressures for the reaction, and the use of expensive solvents. Thus, biotechnological approach has been explored as an alternative. A biotransformation reaction using nitrilase has been used for 4HPAA production [2]. Koma et al. [1] engineered an Escherichia coli for the production of 4HPAA from glucose. Overexpression of the indole-3-pyruvate/phenylpyruvate decarboxylase gene ipdC from Azospirillum brasilense NBRC102289, and the phenylacetaldehyde dehydrogenase gene $f e a B$ from $E$. coli in a tyrosine overproducing $E$. coli strain resulted in the production of $6.1 \mathrm{mM}(0.93 \mathrm{~g} / \mathrm{L})$ 4HPAA with a yield of $13.2 \%(\mathrm{~mol} / \mathrm{mol})$ in a shake flask culture. However, the titer of 4HPAA in engineered microorganisms is much lower than that of other aromatic compounds [13, 14]. Thus, further work is required to increase the production of 4HPAA.

Quorum-sensing (QS) systems has been considered as an auto-induction system that is regulated by cell density. The Lux and Esa QS systems are the main QS systems that have been reported to date. Recently, an Esa QS system from Pantoea stewartii has been engineered to automatically downregulate the competing pathway, significantly improving the production of myo-inositol, glucaric acid and shikimic acid [15].

In this study, we first compared different biosynthetic pathways of 4HPAA (Fig. 1). Then, the biosynthetic pathway enzymes of 4HPAA were directedly evolved and mediated using a tunable intergenic region (TIGR) sequence. Finally, the TIGR-mediated biosynthetic pathway was dynamically regulated using a quorum-sensing circuit. The resulting $E$. coli produced $17.39 \pm 0.26 \mathrm{~g} / \mathrm{L}$
4HPAA without addition of external inducers, which is the highest value reported to date.

\section{Methods}

Strains, plasmids and primers

The bacterial strains, plasmids, and primers used in this study are listed in Table 1. E. coli DH5 $\alpha$ was used for gene cloning in this study. The L-DOPA overproducing strain E. coli DOPA-30N [16] was used as the host strain for the production of 4HPAA.

\section{Construction of plasmids}

The codon-optimized ipdC (GenBank accession number X99587) from A. brasilense NBRC102289, tydc (encoding tyrosine/DOPA decarboxylase, GenBank accession number AE015451) from Pseudomonas putida KT2440, and aas (encoding aromatic aldehyde synthase, GenBank accession number M96070) from Petroselinum crispum (encoding aromatic aldehyde synthase) were synthesized by GENEWIZ, Inc. (Suzhou, China). The $f e a B$ (encoding phenylacetaldehyde dehydrogenase) and $\operatorname{tyn} A$ (encoding tyramine oxidase) genes were amplified from E. coli. The ARO10 (encoding phenylpyruvate decarboxylase) was amplified from Saccharomyces cerevisiae. The protein fusion is a common approach of substrate channeling for coordinating expression of cascade enzymes [19-21]. Thus, a linker encoding (GSG) ${ }_{2}$ was inserted into the two genes, and the TAA stop codon of the front gene was deleted. The fusion pathway was cloned into pBbB2kGFP to obtain pBbB2k-ipdC-L-feaB, pBbB2k-ARO10-LfeaB, pBbB2k-tydc-L-tynA-L-feaB, pBbB2k-aas-L-feaB, respectively.

\section{Generation of random mutagenesis libraries using error-prone PCR and screening}

MEGAWHOP-PCR [22] was performed for the construction of libraries. The random mutagenesis libraries of the fusion ARO10-feaB gene cluster were constructed through error-prone PCR. Primer pairs AROF/FeaR were used for amplification the ARO10-feaB gene cluster using pBbB2k-ARO10-L-feaB as a template. The PCR reaction mixture $(50 \mu \mathrm{L})$ consisted of $5 \mathrm{mM} \mathrm{MgCl}$, $0.3 \mathrm{mM} \mathrm{MnCl} 2,0.2 \mathrm{mM}$ each of dATP, dGTP, dCTP and $\mathrm{dTTP}$, and $2.5 \mathrm{U}$ of rTaq DNA polymerase. The PCR products were then used as megaprimers to perform MEGAWHOP-PCR using pBbB2k-ARO10-L-feaB as a template. Following the MEGAWHOP-PCR, $D p n \mathrm{I}$ digestion $(20 \mathrm{U})$ of the surplus template in the PCR reaction mixture was performed at $37{ }^{\circ} \mathrm{C}$ overnight, then $D p n \mathrm{I}$ was inactivated at $80{ }^{\circ} \mathrm{C}$ for $20 \mathrm{~min}$. The PCR products were transformed into E. coli DH5a cells, plated on LB agar, and then pooled together after overnight growth to create a liquid library. 


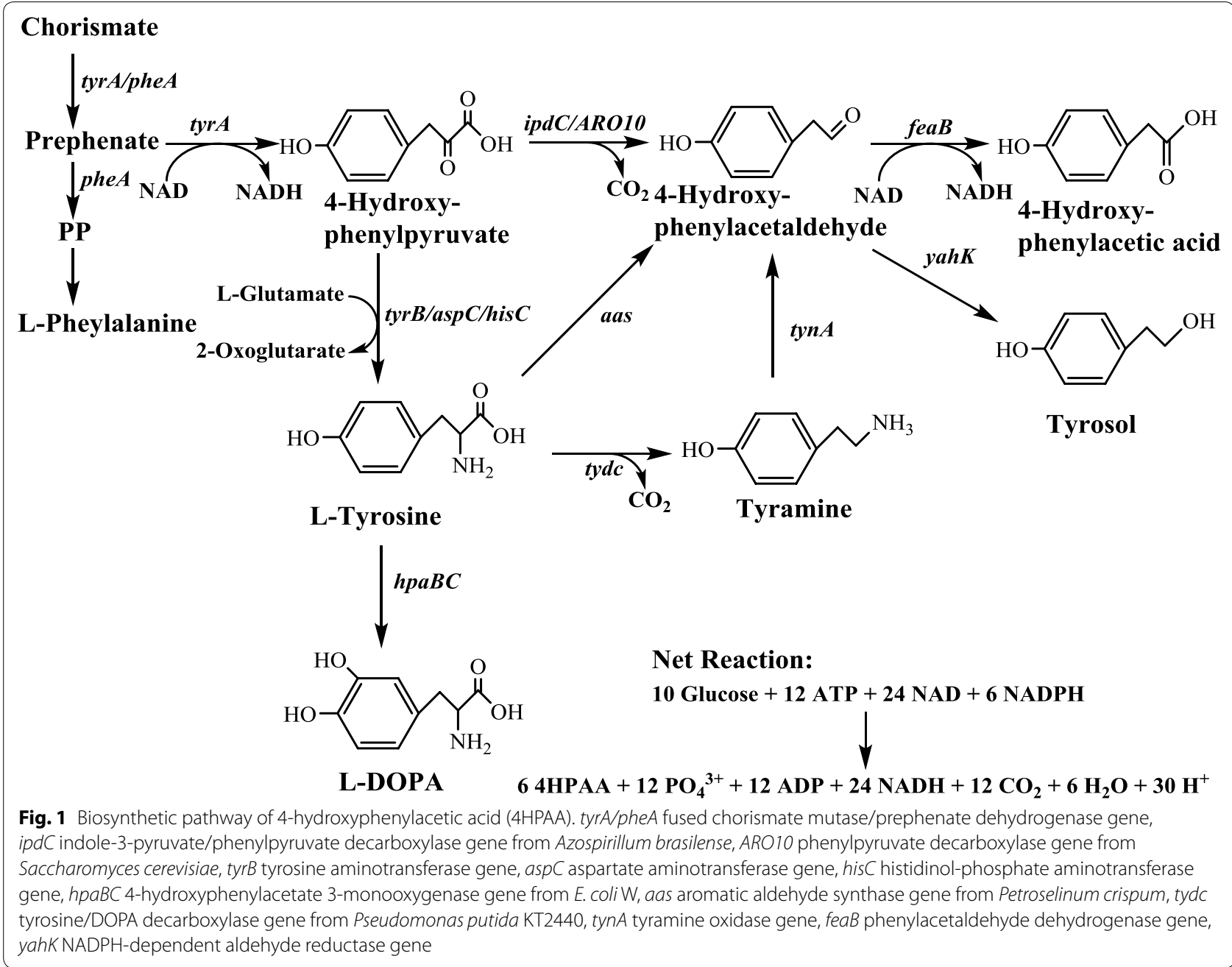

The mutant plasmid was recovered from the library and transferred into the L-DOPA overproducer $E$. coli DOPA$30 \mathrm{~N}$. Then, the resulting transformants were plated on $\mathrm{LB}$ agar with $50 \mathrm{mg} / \mathrm{mL}$ kanamycin and $120 \mathrm{ng} / \mathrm{mL}$ anhydrotetracycline. After overnight growth, individual colonies were inoculated into a 48-well deep-well microplate $(4.6 \mathrm{~mL})$ containing $1 \mathrm{~mL}$ of the fermentation medium and then incubated at $37^{\circ} \mathrm{C}$ and $1000 \mathrm{rpm}$ for $72 \mathrm{~h}$ on a MBR-420FL shaker (TAITEC Corporation, Saitamaken, Japan). Because L-DOPA can be easily oxidized to dopachrome and then polymerized non-enzymatically to form melanin, the culture broth of the host strain E. coli DOPA-30N became black [16]. Because 4HPAA shares the same precursor (tyrosine) with L-DOPA, light color cultures were selected for further shake flask analysis of 4HPAA production.

\section{Creating TIGR libraries and screening}

To replace the linker between $\mathrm{ARO} 10^{*}$ and $f e a B^{*}$ in pBbB2k-ARO10*-L-feaB* with a two-restriction enzyme sequence, the $A R O 10^{*}$ and $f e a B^{*}$ genes were amplified from pBbB2k-ARO10*-L-feaB*. The two genes were then cloned into pBbB2k-GFP to obtain pBbB2k-ARO10"-feaB".

TIGRs were synthesized using PCR to assemble the oligonucleotides into chimeric DNA sequences as described by Pfleger et al. [23] and Li et al. [19]. The assembled products were purified using a nucleotide removal column, amplified using the end-specific primers TIGRs$\mathrm{F}(\mathrm{X}) / \mathrm{TIGRs}-\mathrm{R}(\mathrm{A})$, and then cloned into the NheI/Not I sites of pBbB2k-ARO10*-feaB* to obtain the plasmid libraries pBbB2k-ARO10*-TIGRs-feaB*. The plasmid libraries were transferred into component DOPA-30N to generate the mutant library.

The TIGR library was plated on LB agar plates containing $50 \mu \mathrm{g} / \mathrm{mL}$ kanamycin and $120 \mathrm{ng} / \mathrm{mL}$ anhydrotetracycline. The plates were then incubated at $30{ }^{\circ} \mathrm{C}$ overnight. Single colonies were inoculated into a 48 -well deep-well microplate $(4.6 \mathrm{~mL})$ containing $1 \mathrm{~mL}$ of fermentation medium, and then incubated at $30{ }^{\circ} \mathrm{C}$ and $1000 \mathrm{rpm}$ for 
Table 1 Strains, plasmids and primers used in this study

\begin{tabular}{|c|c|c|}
\hline Name & Description & Source/purpose \\
\hline \multicolumn{3}{|l|}{ Strain } \\
\hline E. coli DH5a & supE44 $\triangle$ (lacZYA-argF) U169 (\$80lacZ $\triangle \mathrm{M} 15)$ hsdR17 recA endA1 gyrA96 thi-1 relA1 & Invitrogen \\
\hline E. coli BW 25113 & $\operatorname{lac}^{19} \mathrm{rrn}_{\mathrm{T} 14} \Delta \mathrm{Ilac}_{\mathrm{W} 16} h s d R 514 \Delta \operatorname{araBAD}_{\mathrm{AH} 33} \Delta r h a B A D_{\mathrm{LD} 78}$ & [17] \\
\hline E. coli DOPA-30N & L-DOPA overproducer & [16] \\
\hline \multicolumn{3}{|l|}{ Plasmid } \\
\hline $\mathrm{pBbB} 2 \mathrm{k}-\mathrm{GFP}$ & $\begin{array}{l}\text { Expressing plasmid, BglBrick vectors, tet promoter, pBBR1 ori, kan', Addgene plasmid } \\
\# 35345\end{array}$ & [18] \\
\hline pBbB2k-ipdC-L-feaB & $\begin{array}{l}\text { pBbB2k derivatives harboring the fusion gene cluster of ipdC from Azospirillum brasi- } \\
\text { lense and feaB from E. coli with a }(\mathrm{GSG})_{2} \text { linker }\end{array}$ & This study \\
\hline pBbB2k-ARO10-L-feaB & $\begin{array}{l}\text { pBbB2k derivatives harboring the fusion gene cluster of } A R O 10 \text { from Saccharomyces } \\
\text { cerevisiae and feaB from E. coli with a }(\mathrm{GSG})_{2} \text { linker }\end{array}$ & This study \\
\hline pBbB2k-aas-L-feaB & $\begin{array}{l}\text { pBbB2k derivatives harboring the fusion gene cluster of aas from Petroselinum crispum } \\
\text { and feaB from E. coli with a (GSG) }{ }_{2} \text { linker }\end{array}$ & This study \\
\hline pBbB2k-tydc-L-tynA-L-feaB & $\begin{array}{l}\text { pBbB2k derivatives harboring the fusion gene cluster of tydc from Pseudomonas putida } \\
\text { KT2440, tynA and feaB from E. coli with a (GSG) } 2 \text { linker }\end{array}$ & This study \\
\hline pBbB2k-ARO10*- L-feaB* & $\begin{array}{l}\text { pBbB2k derivatives harboring the fusion gene cluster of the evolved } A R O 10_{6 D 5} \text { from } S \text {. } \\
\text { cerevisiae and feaB }{ }_{2 E 1} \text { from E. coli with a }(\mathrm{GSG})_{2} \text { linker }\end{array}$ & This study \\
\hline pBbB2k-ARO10*-feaB* & $\begin{array}{l}\text { pBbB2k derivatives harboring the gene cluster of the evolved } A R O 10_{6 D 5} \text { from S. cerevi- } \\
\text { siae and feaB }{ }_{2 E 1} \text { from E. coli, one operon }\end{array}$ & This study \\
\hline pBbB2k-ARO10*-TIGR-feaB* & $\begin{array}{l}\text { pBbB2k derivatives harboring the TIGR-mediated gene cluster of the evolved } A R O 10_{6 D 5} \\
\text { from S. cerevisiae and feaB }{ }_{2 E I} \text { from E. coli }\end{array}$ & This study \\
\hline pZBK & BglBrick/ePathBrick expression vector, pBBR1 ori, P37 promoter, kan' & [19] \\
\hline pZBK-P esas $_{1} I C-P_{\text {esaR }} A S$ & Quorum-sensing plasmid, pBBR1 ori, P37 promoter, kan' & This study \\
\hline pZBK-P ${ }_{\text {esar }}$ AS-4HPAA & $\begin{array}{l}\text { PZBK-P } P_{\text {esas }} I C-P_{\text {esaR }} A S \text { harboring the TIGR-mediated gene cluster of the evolved } A R O 10_{6 D 5} \\
\text { from S. cerevisiae and fea } B_{2 E 1} \text { from E. coli }\end{array}$ & This study \\
\hline \multicolumn{3}{|c|}{ 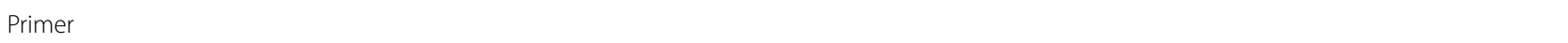 } \\
\hline FeaBF & CCGGAATTCAAAAGATCTGGTATGACAGAGCCGCATGTAGCAG (ECoRI, Bg/ll) & PCR for feaB \\
\hline FeaBR & CGCGGATCCTTAATACCGTACACACACCGACTTAGTTTCACACCAAC (BamHI) & \\
\hline TynAF & CCGGAATTCAAAAGATCTGGTATGGGAAGCCCCTCTCTGTATTCTG (ECORI, Bg/II) & PCR for tynA \\
\hline TynAR & CGCGGATCCACCTGAACCCTTATCTTTCTTCAGCGCCC (BamHI) & \\
\hline ARO10F & $\begin{array}{l}\text { CCGGAATTCAAAAGATCTTTTCGGAATTAAGGAGGTAATAAATATGGCACCTGTTACAATTG } \\
(\text { ECoRI, Bg/II) }\end{array}$ & PCR for $A R 010$ \\
\hline ARO10R & CGCGGATCCACCACTACCTTTTTTATTTCTTTTAAGTG (BamHI) & \\
\hline AROF & GATAGAGAAAAGAATTCAAAAGATCTTTTCGGAATTAAGGAGG (ECoRl, Bg/II) & error-prone PCR for $A R O 10$-feaB \\
\hline FeaR & TACTCGAGTTTGGATCCTTAATACCGTACACACACCGACTT (BamHI) & \\
\hline epAROF & ACCTTCGATTCCGACCTCATTAAGC & error-prone PCR for $A R O 10$ \\
\hline epAROR & CGCGGATCCTTAATACCGAACACACACCGACTTAGTTTCACACCAAC & \\
\hline TIGRs-F(X) & CTAGCTAGCGCCTAGCAAGATCTCCTGATCCCGGTGC (Nhel) & PCR for TIGRs \\
\hline TIGRs-R(A) & TTTTCCTTTTGCGGCCGCGGATACAGTATCTGCGGTACCCTAG (Notl) & \\
\hline Qaro10F & CGACACCTTGTTTCCAACCG & qRT-PCR for ARO10 \\
\hline Qaro10R & TGAAGTCACCAGGAACACCG & \\
\hline QfeaBF & GCCAACGCTGGTGGTAAATC & qRT-PCR for feaB \\
\hline QfeaBR & GCCTCTTCTCCATCCGCTAC & \\
\hline
\end{tabular}

$72 \mathrm{~h}$ on a MBR-420FL shaker (TAITEC, Japan). Lighter color cultures were selected for further shake flask analysis of 4HPAA production.

\section{Construction of a quorum-sensing circuit}

The quorum-sensing fragment containing the signal molecule acyl-homoserine lactone (AHL) synthase gene
esaI, the transcriptional regulator esaR $R^{I 70 V}$, and $\mathrm{P}_{\text {easS }}$ and $\mathrm{P}_{\mathrm{esa}}$ promoters was synthesized by GENEWIZ, Inc (Suzhou, China) and then cloned into the MluI/SalI of pZBK [19] to obtain the plasmid pZBK-P ${ }_{\text {easS }}$ IC-P easR $_{\text {AS }}$ (Additional file 1: Figure S1A). The TIGR-mediated gene cluster of the evolved $A R O 10$ and $f e a B$ was cloned 
into the multiple cloning site 2 (MCS2) of pZBK$\mathrm{P}_{\text {eass }} \mathrm{IC}-\mathrm{P}_{\text {easR }}$ AS to obtain pZBK- $\mathrm{P}_{\text {esar }}$ AS-4HPAA.

\section{Production of 4HPAA}

A single colony was inoculated into $5 \mathrm{~mL}$ of LB medium in a falcon tube then cultured overnight at $30{ }^{\circ} \mathrm{C}$. The overnight seed culture was then inoculated into $50 \mathrm{~mL}$ of fermentation medium with a starting $\mathrm{OD}_{600}$ of 0.1 . The fermentation medium contained (per liter) tryptone $(10 \mathrm{~g})$, yeast extract $(5 \mathrm{~g}), \mathrm{NaCl}(10 \mathrm{~g})$, glucose $(40 \mathrm{~g})$, $\mathrm{KH}_{2} \mathrm{PO}_{4}(0.6 \mathrm{~g}), \mathrm{Na}_{2} \mathrm{HPO}_{4} \cdot 7 \mathrm{H}_{2} \mathrm{O}(2.56 \mathrm{~g})$, and $10 \mathrm{~mL}$ of trace element solution. The trace element solution contained (per liter) $\mathrm{FeSO}_{4} \cdot 7 \mathrm{H}_{2} \mathrm{O}(10 \mathrm{~g}), \mathrm{ZnSO}_{4} \cdot 7 \mathrm{H}_{2} \mathrm{O}$ $(2.2 \mathrm{~g}), \quad \mathrm{MnSO}_{4} \cdot 4 \mathrm{H}_{2} \mathrm{O}(0.58 \mathrm{~g}) \mathrm{CuSO}_{4} \cdot 5 \mathrm{H}_{2} \mathrm{O}(1 \mathrm{~g})$, $\left(\mathrm{NH}_{4}\right)_{6} \mathrm{Mo}_{7} \mathrm{O}_{24} \cdot 4 \mathrm{H}_{2} \mathrm{O}(0.1 \mathrm{~g})$, and $\mathrm{Na}_{2} \mathrm{~B}_{4} \mathrm{O}_{7} \cdot 10 \mathrm{H}_{2} \mathrm{O}(0.2 \mathrm{~g})$. The $\mathrm{pH}$ of the medium was adjusted to 7.0. The main cultures were incubated at $30{ }^{\circ} \mathrm{C}$ and $200 \mathrm{rpm}$. When the $\mathrm{OD}_{600}$ reached 2.5, the cultures were induced with $120 \mathrm{ng} / \mathrm{mL}$ anhydrotetracycline. After induction, the cultures were incubated at $30^{\circ} \mathrm{C}$ and $200 \mathrm{rpm}$ for $72 \mathrm{~h}$.

Fed-batch fermentation was carried out in a $2 \mathrm{~L}$ bioreactor (MiniBox 2L*2 Parallel Bioreactor System, T\&J Bio-engineering (Shanghai) Co. LTD, Shanghai, China) containing $1.2 \mathrm{~L}$ of fermentation medium with an initial $\mathrm{OD}_{600}$ of approximately 0.1 . Fermentation was carried out at $30{ }^{\circ} \mathrm{C}$ with an airflow of $1.2 \mathrm{~L} / \mathrm{min}$ and a starting agitation rate of $400 \mathrm{rpm}$. For $E$. coli containing the statically controlled pathway, $120 \mathrm{ng} / \mathrm{mL}$ anhydrotetracycline was added as an inducer after $6 \mathrm{~h}$. The $\mathrm{pH}$ was controlled at 7.0 by automatic addition of $\mathrm{NH}_{4} \mathrm{OH}$. Dissolved oxygen was maintained above $25 \%$ by adjusting the agitation rate. The feed solution ( $\mathrm{pH} 7.0$,) contained $500 \mathrm{~g} / \mathrm{L}$ glucose and $30 \mathrm{~g} / \mathrm{L} \mathrm{MgSO}_{4} \cdot 7 \mathrm{H}_{2} \mathrm{O}$. The feed was introduced continuously into the fermenter using a $\mathrm{pH}$-stat feeding strategy. Once the glucose was exhausted, the $\mathrm{pH}$ rose rapidly. When the $\mathrm{pH}$ was greater than 7.0 by 0.1 of a $\mathrm{pH}$ unit, the feed was automatically added to the fermenter. Samples were periodically withdrawn, and the following parameters were measured: $\mathrm{OD}_{600}$, residual glucose concentrations, and 4HPAA concentrations. Fermentation experiments were performed in duplicate.

\section{Catalytic efficiency assays}

For in vitro activity assay, E. coli DOPA-30N harboring pBbB2k-ARO10-L-feaB or pBbB2k-ARO10"-L-feaB* were pre-inoculated in $5 \mathrm{~mL}$ of LB medium overnight and then inoculated into $50 \mathrm{~mL}$ the fermentation medium containing kanamycin. The cultures were incubated at $30{ }^{\circ} \mathrm{C}$ and $200 \mathrm{rpm}$ until $\mathrm{OD}_{600}$ reached 2.5 and then induced with $120 \mathrm{ng} / \mathrm{mL}$ anhydrotetracycline. After $44 \mathrm{~h}$, the culture broth was disrupted directly using an Ultrahigh Pressure Cell Disrupter (JN-3000Plus, Guangzhou Juneng Nano \& Bio Technology Co. LTD, China), and then centrifuged to remove cell debris. The supernatant was selected as a crude enzyme solution for catalytic efficiency assay. The total protein concentration in the crude extract was determined by a Bradford assay using a BioRad protein assay kit (Bio-Rad Laboratories, Hercules, CA, USA). The catalytic efficiency assay was conducted as follows: 4-hydroxyphenylpyruvic acid of $10 \mathrm{mM}$ was added to the crude enzyme. The reaction was conducted at $37^{\circ} \mathrm{C}$ for $6 \mathrm{~h}$. Then, the reaction mixture was immediately placed on ice broth to stop the reaction. The product (4HPAA) concentration in the reaction solution was determined immediately by HPLC.

For in vivo activity assay, E. coli BW25113 was transformed with pBbB2k-ARO10-L-feaB and pBbB2kARO10*-L-feaB*, respectively. Single colonies were pre-inoculated into $5 \mathrm{~mL}$ of LB medium containing kanamycin and cultured overnight at $30{ }^{\circ} \mathrm{C}$ and $200 \mathrm{rpm}$. A $500 \mu \mathrm{L}$ overnight cultures were inoculated into $50 \mathrm{~mL}$ the fermentation medium containing kanamycin. The cultures were incubated at $30^{\circ} \mathrm{C}$ and $200 \mathrm{rpm}$ until $\mathrm{OD}_{600}$ reached 2.5 and then induced with $120 \mathrm{ng} / \mathrm{mL}$ anhydrotetracycline at $37^{\circ} \mathrm{C}$ and $200 \mathrm{rpm}$. Three hours after induction, 4-hydroxyphenylpyruvic acid was added to a final concentration of $1.0 \mathrm{~g} / \mathrm{L}$ and incubated at $30{ }^{\circ} \mathrm{C}$ and $200 \mathrm{rpm}$ for $6 \mathrm{~h}$. Growth and the concentration of 4HPAA were analyzed.

\section{Assay}

Growth was monitored by measuring the optical density at $600 \mathrm{~nm}$. 4HPAA in the supernatants was analyzed using a Shimadzu HPLC system (LC-20A, Shimadzu, Japan) equipped with an Inertsil ODS-SP column $(5 \mu \mathrm{m}$, $4.6 \times 150 \mathrm{~mm}$, GL Sciences Inc., Tokyo, Japan). The mobile phase was $0.2 \%$ TFA in methanol, and the flow rate was $0.5 \mathrm{~mL} / \mathrm{min}$ at $30{ }^{\circ} \mathrm{C}$. The methanol concentration was increased from 14 to $45 \%$ for $20 \mathrm{~min}$, and then decreased to $14 \%$. This concentration was then maintained for $10 \mathrm{~min}$. A photodiode array detector (SPDM20A) operating at $222 \mathrm{~nm}$ was used, and a standard curve was constructed from serial dilutions of a standard stock solution. Glucose concentrations were determined using glucose oxidase and a glucose assay kit (Shanghai Rongsheng Biotech Corporation, Shanghai, China).

\section{Statistical analysis}

All experiments were conducted in triplicate, and the data were averaged and presented as the mean \pm standard deviation. One-way analysis of variance followed by Tukey's test was used to determine significant differences using the OriginPro (version 7.5) package. Statistical significance was defined as $p<0.05$. 


\section{Results and discussion}

\section{Screening biosynthetic pathways}

Three biosynthetic pathways (Fig. 1) have been developed for the production of tyrosol; namely, the Ehrlich pathway via the ipdC [1] and the yeast $A R O 10$ $[24,25]$, the aas (encoding aromatic aldehyde synthase) pathway [26] and the tydc-tyo (encoding tyrosine decarboxylase and tyramine oxidase, respectively) pathway [27]. Because 4HPAA shares the same precursor, 4-hydroxyphenylacetaldehyde (4HPAAL), with tyrosol, we first constructed these pathways and evaluated their 4HPAA productions (Fig. 1). These pathways were then introduced into the L-DOPA overproducing $E$. coli strain DOPA-30N for shake flask analysis of 4HPAA. No L-DOPA was detected in the four engineered strains, indicating that, the rate of 4HPAA production was higher than that for L-DOPA (data not shown). Thus, E. coli DOPA-30N was used as the parent strain for the production of 4HPAA in this study. The introduction of all pathways resulted in the production of 4HPAA (Table 2), indicating that these pathways can be used for 4HPAA production. Moreover, the yeast Ehrlich pathway produced the highest titer of 4HPAA (Table 2). The recombinant strain harboring the yeast Ehrlich pathway produced $3.08 \pm 0.05 \mathrm{~g} / \mathrm{L}$ 4HPAA with a molar yield of $13.4 \%$. The same result was obtained for tyrosol. The yeast Ehrlich pathway also exhibited the highest biosynthetic efficiency for tyrosol production among the four pathways [1, 24-27]. Therefore, the yeast Ehrlich pathway was selected as the biosynthetic pathway for the production of 4HPAA in this study.

Although only ipdC Ehrlich pathway was reported for the production of 4HPAA in literatures, our results demonstrated that three other pathways based on the reported tyrosol biosynthetic pathways can be used for the production of 4HPAA. Moreover, the 4HPAA titer that was obtained via our yeast Ehrlich pathway was 1.33-fold as high as that obtained via the ipdC Ehrlich pathway.
Directed evolution of the biosynthetic pathway Independent of the enzyme structure and catalytic mechanism, directed evolution of enzyme is widely used to alter the coding sequence and improve the catalytic activities of enzymes. Thus, to increase the efficiency of the biosynthetic pathway, the fusion gene cluster of ARO10$f e a B$ was directedly co-evolved by error-prone PCR. L-DOPA can be easily oxidized to dopachrome and then polymerized non-enzymatically to form the black pigment melanin [28]. Because the preparation of 4HPAA utilizes the same precursors (4-hydroxyphenylpyruvate/ tyrosine) as L-DOPA (Fig. 1), 4HPAA biosynthesis competes with L-DOPA. The higher the activity of the fusion gene cluster, the lower the production of L-DOPA, hence, the culture broth becomes lighter. A total of 792 colonies were used for the assay of 4HPAA production in deepwell microplate cultures. Twenty-three colonies with lighter colors were selected for further shake flask analysis. As shown in Fig. 2a, strains no. 12 and 23 produced the highest level of $4 \mathrm{HPP}(4.66 \pm 0.14$ and $4.65 \pm 0.10 \mathrm{~g} / \mathrm{L}$, respectively). These mutant plasmids, denoted as $2 \mathrm{E} 1$ and 4F3, were isolated and sent for sequencing. The sequencing results are presented in Additional file 1: Table S1. In plasmid 2E1, one base mutant (C1869T) in ARO10 and three base mutants (T72G, T1195C, and A1491T) were observed in $f e a B$. In plasmid 4F3, two base mutants (A1682G and A1842G) in ARO10 and three base mutants (C165G, A609T, and T1296) were observed in feaB. None of the base mutants in ARO1O produced an amino acid change. One amino acid mutant of $f e a B$ was observed in plasmid 2E1 (I24M) and 4F3 (N55K).

Because no amino acid mutant in $A R O 10$ was observed after the co-evolution of the $A R O 10-f e a B$, the $A R O 10$ in pBbB2k-ARO10-L-feaB was single-evolved using error-prone PCR. Of 900 colonies used for the assay in deep-well microplate cultures, twelve colonies with lighter colors were selected for further shake flask analysis. As shown in Fig. 2b, strains no. 6, 11, 13, and 15 produced higher levels of 4HPAA than the wildtype strain. The mutant plasmids, denoted as 6D5, 9F5, $10 \mathrm{~A} 3$, and 10G5, were isolated and sent for sequencing. DNA sequencing revealed some base mutations

Table 2 4-Hydroxyphenylacetic acid production in E. coli DOPA-30N harboring different biosynthetic pathways

\begin{tabular}{|c|c|c|c|c|}
\hline Host strain & Plasmid & OD600 & Titer (g/L) & Yield (\%, $\mathrm{mol} / \mathrm{mol})$ \\
\hline \multirow[t]{6}{*}{ E. coli DOPA-30N } & pBbB2k-ipdC-L-feaB & $16.28 \pm 0.45$ & $2.32 \pm 0.05$ & $11.8 \pm 0.2$ \\
\hline & pBbB2k-ARO10-L-feaB & $17.36 \pm 0.19$ & $3.08 \pm 0.05$ & $13.4 \pm 0.2$ \\
\hline & pBbB2k-aas-L-feaB & $17.13 \pm 1.44$ & $2.11 \pm 0.06$ & $9.3 \pm 0.2$ \\
\hline & pBbB2k-tydC-L-tynA-L-feaB & $15.44 \pm 0.42$ & $2.41 \pm 0.04$ & $12.1 \pm 0.2$ \\
\hline & pBbB2k-ARO10*-L-feaB* & $16.68 \pm 0.35$ & $5.64 \pm 0.06$ & $22.9 \pm 0.3$ \\
\hline & pBbB2k-ARO10*-TIGR-feaB* & $17.12 \pm 0.32$ & $6.58 \pm 0.16$ & $27.5 \pm 0.3$ \\
\hline
\end{tabular}



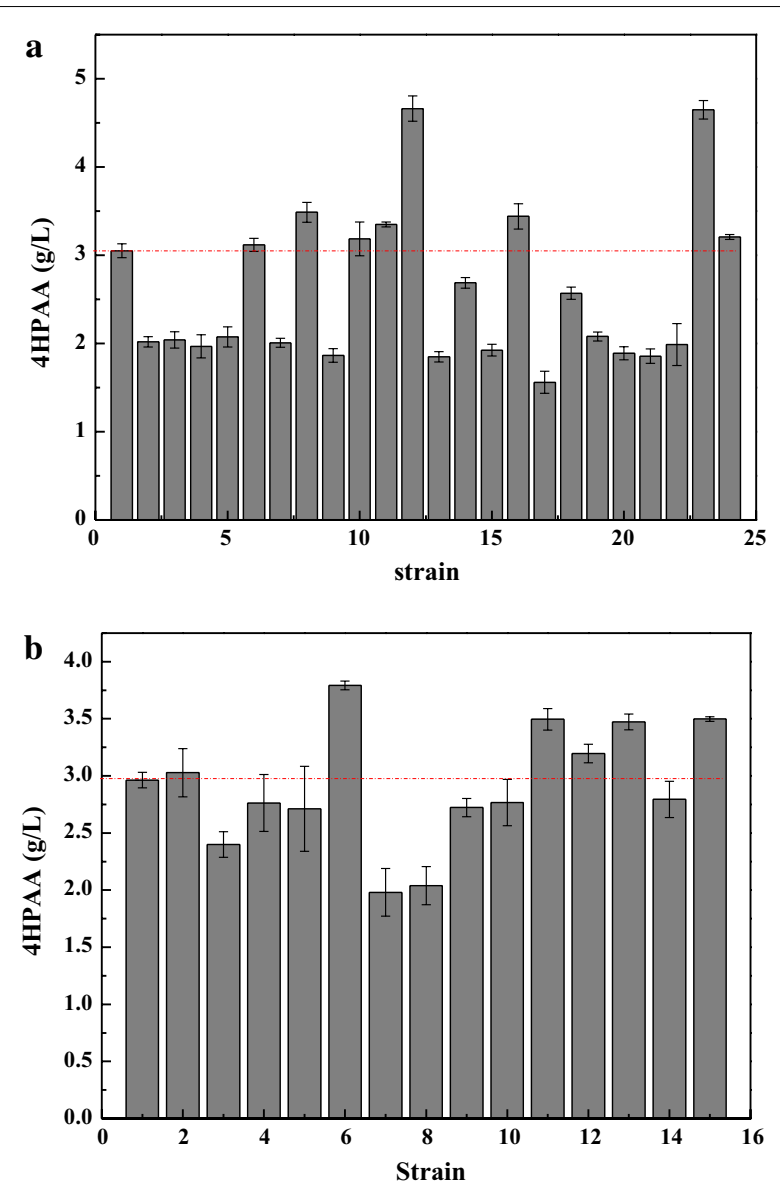

Fig. 2 4HPAA production by E. coli DOPA-30N harboring mutant gene clusters from error-prone PCR. a The mutant gene clusters caused by the co-evolution of the ARO10-L-feaB. $\mathbf{b}$ The mutant gene clusters caused by the single-evolution of $A R O 10$. Cells were grown at $30^{\circ} \mathrm{C}$ and $200 \mathrm{rpm}$ for $72 \mathrm{~h}$. See "Methods" for other details. E. coli DOPA-30N (pBbB2k-ARO10-L-feaB) (strain no. 1) was set the control. The data represent the means of three replicates, and error bars represent standard deviations

and amino acid mutations in ARO10 (Additional file 1: Table S1). Two amino acid mutations (F138L and D218G) were observed in plasmid 6D5, and one mutation (V451I) was observed in plasmid 10A3.

To investigate the effects of these mutated genes on the production of 4HPAA, we combined these mutated genes to create a biosynthetic pathway and introduced the pathway into E. coli DOPA-30N. Figure 3 shows that mutations of two genes indeed increased the production of 4HPAA. The engineered strain harboring both mutated genes produced higher levels of 4HPAA than the strain that contained only one mutated gene. The combination of the mutated ARO10 from plasmid $6 \mathrm{D} 5$ with the mutated $f e a B$ from plasmid $2 \mathrm{E} 1$ resulted in the highest titer of 4HPAA $(5.64 \pm 0.06 \mathrm{~g} / \mathrm{L})$. This

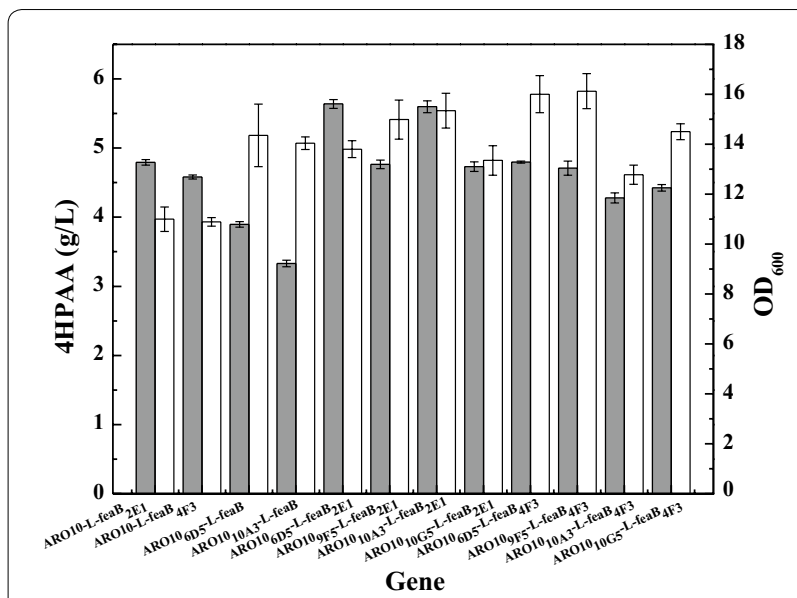

Fig. 3 4HPAA production by E. coli DOPA-30N harboring the fusion gene cluster of different combinations of the evolved S. cerevisiae $A R O 10$ and E. coli feaB. Growth (white bar); 4HPAA concentration (gray bar). Cells were grown at $30^{\circ} \mathrm{C}$ and $200 \mathrm{rpm}$ for $72 \mathrm{~h}$. See "Methods" for other details. The data represent the means of three replicates,

and error bars represent standard deviations

combination resulted in approximately a $90 \%$ increase in the production of 4HPAA compared to the wild-type genes.

To evaluate the applicability of the evolved pathway enzymes in microbial production of 4HPAA, we first performed in vitro crude extract enzyme assays to measure their catalytic activities. 4-Hydroxyphenylpyruvic acid of $10 \mathrm{mM}$ was added into the cell crude extracts of $E$. coli DOPA-30N harboring the wild-type or mutant plasmid, respectively. The catalytic efficiencies were measured by testing the 4HPAA formation rate. As shown in Fig. 4, directed evolution resulted in an increase in the in vitro catalytic efficiency of the pathway to $45.55 \pm 2.43 \mathrm{mg} / \mathrm{h} / \mathrm{g}$ protein from $23.67 \pm 2.66 \mathrm{mg} / \mathrm{h} / \mathrm{g}$ protein (Fig. 4), indicating that the evolved pathway was more efficient at converting 4-hydroxyphenylpyruvate to 4HPAA than the wild-type pathway in vitro.

Then, the initial in vivo efficiency of converting 4-hydroxyphenylpyruvic acid to 4HPAA was analyzed through whole-cell bioconversion experiments. The results were compared in Fig. 4. The in vivo efficiency of the evolved pathway was $3.28 \pm 0.12 \mathrm{mg} / \mathrm{L} / \mathrm{h} /$ $\mathrm{OD}$, while the in vivo efficiency of the parent pathway was $1.91 \pm 0.05 \mathrm{mg} / \mathrm{L} / \mathrm{h} / \mathrm{OD}$, indicating a $72 \%$ increment in activity level. This result is consistent with the in vitro crude enzyme assays. This result indicates that the evolved pathway was more efficient at converting 4-hydroxyphenylpyruvate to 4HPAA than the wild-type pathway both in vitro and in vivo.

Directed evolution is the most commonly used approach used to significantly improve the performance of pathway enzymes. Applying direction evolution, we 


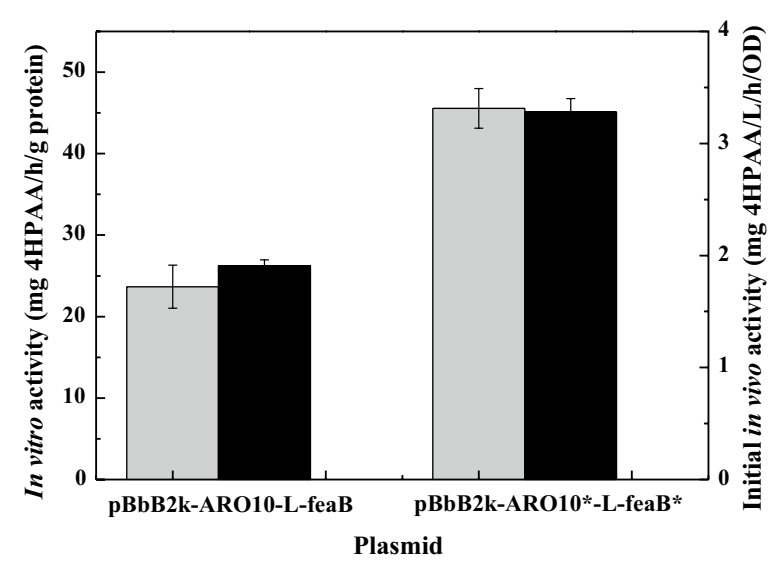

Fig. 4 In vitro (gray bar) and initial in vivo (black bar) catalytic efficiency of the pathway. The data represent the means of three replicates, and error bars represent standard deviations

isolated mutant variants with improved activity than the wild-type enzymes. The best combination of the ARO10 and $\mathrm{FeaB}$ mutants resulted in a $90 \%$ increase in the production of 4HPAA. The increased 4HPAA titer may be as a result of increases in the activity of pathway enzymes.

The unbalanced expression of multiple genes may result in the accumulation of toxic metabolic intermediates, thereby reducing product titers. Thus, Pfleger et al. [23] developed a combinatorial engineering approach for coordinating the expression of cascade enzymes by generating libraries of TIGRs. They applied the TIGR approach to balance gene expression in the MEV pathway, resulting in a sevenfold increase in the production of mevalonate. We also applied the TIGR approach to improve the production of zeaxanthin [19] and pinene [29]. Thus, we constructed a library of TIGRs to balance the expression of the evolved $A R O 10^{*}$ and $f e a B^{*}$. The library of TIGRs was inserted between the two genes. A total of 1080 colonies were used for the assay of 4HPAA production in deep-well microplate cultures. Twenty colonies with lighter colors were selected for further shake flask analysis. As shown in Fig. 5a, strains nos. 4, 10, and 12 produced higher levels of 4HPAA. We again assayed the production of 4HPAA in the three strains (Fig. 5b). Strain no. 12 produced higher levels of 4HPAA, $6.58 \mathrm{~g} / \mathrm{L}$ (Fig. 5b). Regulation of TIGR resulted in a $16.7 \%$ increase in the production of 4HPAA compared with the fusion evolved pathway. The TIGR in strain no. 12 was sequenced and the results are presented in Additional file 1: Table S2.

\section{Dynamic regulation of the biosynthetic pathway}

In the above study, the inducible expression system was used to regulate the biosynthetic pathway of 4HPAA.
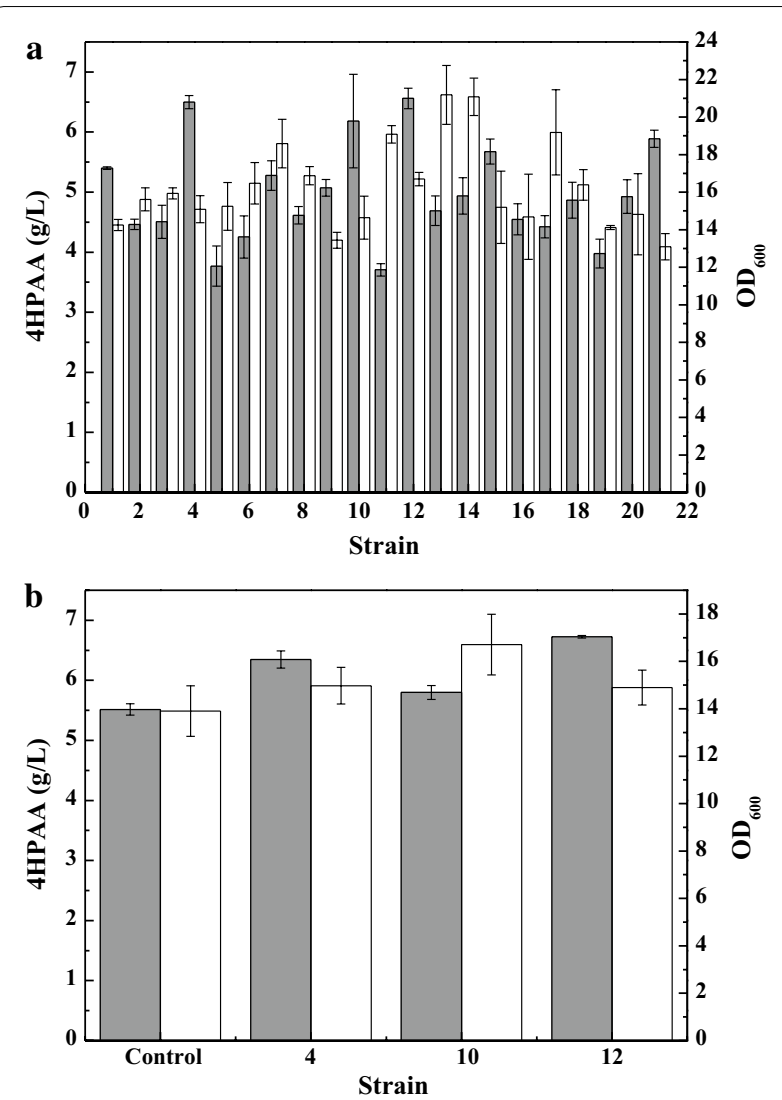

Fig. 5 Growth (white bar) and 4HPAA production (gray bar) for E. coli DOPA-30N harboring the TIGR-mediated gene cluster of the evolved S. cerevisiae $A R O 10^{*}$ and E. coli feaB* a Colonies with lighter colors from the TIGR library; $\mathbf{b}$ strains with higher levels of 4 HPAA. Cells were grown at $30^{\circ} \mathrm{C}$ and $200 \mathrm{rpm}$ for $72 \mathrm{~h}$. See "Methods" for other details. E. coli DOPA-30N (pBbB2k-ARO10*-L-feaB*) was set the control. The data represent the means of three replicates, and error bars represent standard deviations

However, this system has some disadvantages, including leaky expression, lack of dynamic control, and the prohibitively high cost of inducers that are associated with large-scale production. QS functions to control gene expression in response to cell density, and has been applied to several biosynthetic pathways to produce glucaric acid, myo-inositol, shikimate [15], $\beta$-lactamase exoenzyme [30], isopropanol [31], and bisabolene [32] in $E$. coli, and para-hydroxybenzoic acid in Saccharomyces cerevisiae [33]. The Lux and Esa QS systems are the main QS systems that have been reported to date. The QS system requires two regulatory elements, a signaling molecule (also known as an autoinducer) that is constitutively produced, and a receptor protein that binds the autoinducer and acts as a transcriptional activator or repressor. The Esa QS system from P. stewartii, has been engineered for both transcriptional activation and repression by regulating EsaR binding sites on the promoter 
[34]. In the absence of signal molecules, 3-oxohexanoylhomoserine lactone (AHL), the transcriptional regulator in EsaRI70V, binds the $\mathrm{P}_{\text {esaS }}$ or $\mathrm{P}_{\text {esaR }}$ promoter and activates or represses transcription, respectively $[15,34,35]$. Thus, we constructed an Esa QS plasmid, pZBK- $\mathrm{P}_{\text {esaS }} \mathrm{IC}-$ $\mathrm{P}_{\text {esaR }} \mathrm{AS}$, with both activating and repressing functions (Additional file 1: Figure S1A). To characterize the function of the QS circuit, GFP and mCherry genes were successively inserted into the multiple cloning site 1 (MSC1) and MSC2 to obtain pZBK- $\mathrm{P}_{\text {esas }}$-GFP-P $\mathrm{P}_{\text {esaR }}$-mCherry (Additional file 1: Figure S1B). The relative fluorescence of GFP decreased with cell growth (Additional file 1: Figure S1C). From Additional file 1: Figure S1C, we can also see that the relative fluorescence of mCherry increased with cell growth. These results demonstrate that the $\mathrm{P}_{\text {esas }}$-controlled gene was indeed repressed and that the $\mathrm{P}_{\text {esaR }}$-controlled gene was activated by the induction of AHL in response to cell density.

Thus, the TIGR-mediated gene cluster of the evolved $A R O 10_{6 D 5}$ and $f e a B_{2 E 1}$ was cloned into the MCS2 site of the Esa QS plasmid pZBK- $\mathrm{P}_{\text {esas }} \mathrm{IC}-\mathrm{P}_{\text {esaR }} \mathrm{AS}$ to obtain pZBK- $P_{\text {esaR }}$ AS-4HPAA, which was then transferred into E. coli DOPA-30N. In this strain, the pathway was controlled by the $\mathrm{P}_{\text {esar }}$ promoter, indicating that the expression of the pathway was activated by the induction of AHL in response to cell density (Fig. 6a). To compare the production of 4HPAA between the recombinant strain harboring the QS-controlled and the statically controlled pathway, fed-batch cultures were conducted in 2-L bioreactors. The results are presented in Fig. $6 \mathrm{~b}$. The engineered strain harboring the statically controlled pathway produced 4HPAA of $11.88 \pm 0.82 \mathrm{~g} / \mathrm{L}$ with a molar yield of $14.1 \%$ at $84 \mathrm{~h}$. The engineered strain harboring the QS-controlled pathway produced higher level of 4HPAA titer, which achieved $17.39 \pm 0.26 \mathrm{~g} / \mathrm{L}$ with a molar yield of $23.2 \%(\mathrm{~mol} / \mathrm{mol})$ at $76 \mathrm{~h}$. This indicates that QS-regulation increased the production of 4 HPAA by $46.4 \%$ compared to the statically controlled pathway.

A Lux QS system from Vibrio fischeri has been used to construct a toggle switch for redirecting metabolic flux from the TCA cycle to the production of isopropanol, resulting in approximately a threefold increase in the titer [31]. This Lux QS system still requires external IPTG inducer to turn on LuxR/LuxI expression. Recently, an inducer-free Lux QS system has been used to dynamically regulate heterologous mevalonate and bisabolene biosynthetic pathways to improve the inducer-free production of bisabolene by $44 \%$ in $E$. coli [32]. An EsaR-P esas QS system from P. stewartii has been constructed to improve glucaric acid in E. coli by dynamically repressing glycolysis, leading to a fivefold increase in its titer [15]. In their study, the QS system

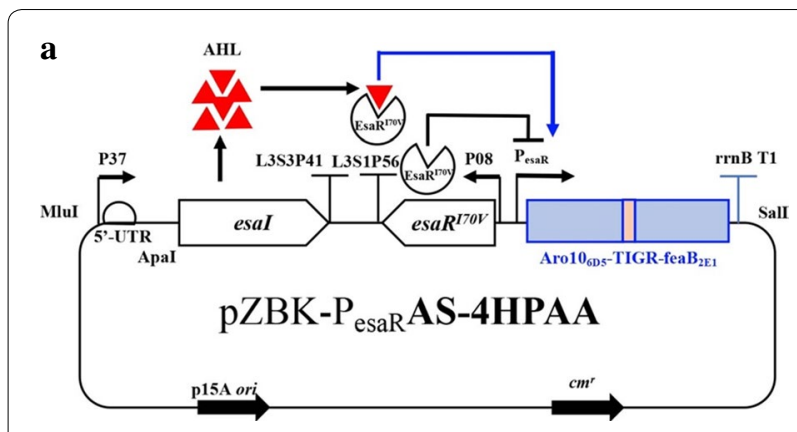

b

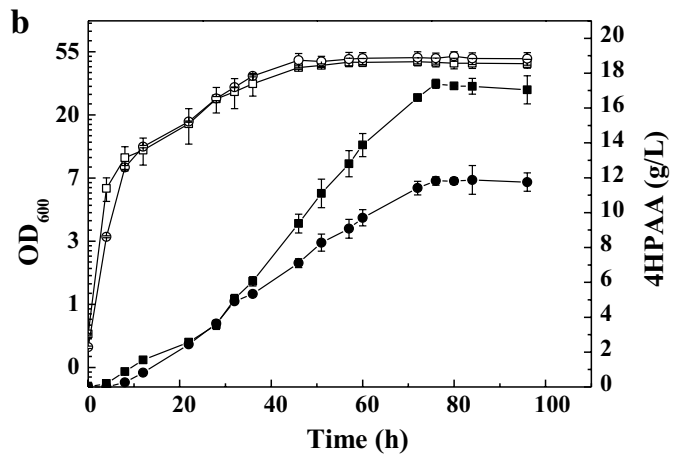

Fig. 6 a The quorum-sensing (QS) system for 4HPAA production. In the absence of 3-oxohexanoyl-homoserine lactone (AHL, red triangle), the transcriptional regulator $\mathrm{EsaR}^{170 \mathrm{~V}}$ binds the $\mathrm{P}_{\text {esaR }}$ promoter and represses the transcription of the pathway enzymes. Esal proteins synthesizes autoinducer AHL, which can diffuse in or out cells and binds to the transcriptional regulator EsaR ${ }^{170 V}$. At the threshold level of AHL, which accumulates in proportion to cell density, the transcriptional regulator $E s a R^{170 V}$ binds to $A H L$, leading to dissociation of EsaR ${ }^{170 V_{\text {from }}}$ the $P_{\text {esaR }}$ promoter, and the EsaR ${ }^{170 V}-A H L$ complex activates the transcription of the pathway enzymes. $\mathbf{b}$ Fed-batch cultures of E. coli DOPA-30N harboring the QS-controlled (squares) and the statically controlled (circles) pathway for 4HPAA production. Growth (open: open square, open circle); 4HPAA concentration (black: filled square, filled circle). Experiments were conducted in duplicate, and measurements are presented as the means with standard deviations

was used to repress the competing pathway, driving the flux toward the biosynthetic pathway. In our study, we constructed an Esa- $\mathrm{P}_{\text {esaR }}$ activation QS system to dynamically control the biosynthetic pathway for the inducer-free production of 4HPAA, leading to a $46.4 \%$ improvement in the titer. This is the first report on an Esa activation QS system for dynamically regulating a biosynthetic pathway. The 4HPAA titer obtained in this study is 18.7 -fold the maximum reported in literatures $(0.93 \mathrm{~g} / \mathrm{L})$.

\section{Conclusion}

We designed three biosynthetic pathways of 4HPAA based on tyrosol synthesis. Of these pathways, the yeast Ehrlich pathway exhibited the highest efficiency for the production of 4HPAA. To increase the 
pathway efficiency, the yeast Ehrlich pathway enzymes were directedly evolved using error-prone PCR. After directed evolution, two $\mathrm{ARO} 10$ and $\mathrm{FeaB}$ variants that outperformed the wild-type enzymes were obtained. These mutations increased the efficiency for converting 4-hydroxyphenylpyruvate to 4HPAA both in vitro and in vivo. The TIGR regulation for the evolved pathway enzymes further improved 4HPAA production. An Esa QS circuit with both activating and repressing functions was developed. The Esa- $\mathrm{P}_{\text {esaR }}$ activation QS system was used to dynamically control the biosynthetic pathway of 4HPAA, which yielded $17.39 \pm 0.26 \mathrm{~g} / \mathrm{L}$ 4HPAA without addition of external inducers. The titer is the highest value that has been obtained to date.

\section{Additional file}

Additional file 1: Figure S1. (A) Esa quorum-sensing (QS) plasmid. (B) Activation and repression of genes using quorum-sensing circuit. Table S1. Mutations on the evolved gene. Table S2. The sequence of the TIGR.

\section{Authors' contributions}

YPS performed all of the experimental works. LSF and ZBY performed the fed-batch fermentation and the assay. JZL designed the study and wrote the manuscript. All authors read and approved the final manuscript.

\section{Acknowledgements}

Not applicable.

\section{Competing interests}

The authors declare that they have no competing interests.

\section{Availability of data and materials}

Not applicable.

\section{Consent for publication}

Not applicable.

\section{Ethics approval and consent to participate}

Not applicable.

\section{Funding}

This work was funded by the National Natural Science Foundation of China (Grant No. 21276289 and 201808248), the Natural Science Foundation of Guangdong Province (No. 2015A030311036 and 2018A030310255), the Project of the Scientific and Technical Program of Guangdong Province (No. 2015A010107004) and the Project of the Scientific and Technical Program of Guangzhou (No. 201607010028) for their financial support.

\section{Publisher's Note}

Springer Nature remains neutral with regard to jurisdictional claims in published maps and institutional affiliations.

Received: 2 January 2019 Accepted: 13 April 2019

Published online: 23 April 2019

\section{References}

1. Koma D, Yamanaka H, Moriyoshi K, Ohmoto T, Sakai K. Production of aromatic compounds by metabolically engineered Escherichia coli with an expanded shikimate pathway. Appl Environ Microb. 2012;78(17):6203-16.

2. Thakur N, Kumar V, Thakur S, Sharma N, Sheetal, Bhalla TC. Biotransformation of 4-hydroxyphenylacetonitrile to 4-hydroxyphenylacetic acid using whole cell arylacetonitrilase of Alcaligenes faecalis MTCC 12629. Process Biochem. 2018;73:117-23.

3. Ng TB, Liu F, Lu YH, Cheng CHK, Wang ZT. Antioxidant activity of compounds from the medicinal herb Aster tataricus. Comp Biochem Phys C. 2003;136(2):109-15.

4. Du L, Mei HF, Yin X, Xing YQ. Delayed growth of glioma by a polysaccharide from Aster tataricus involve upregulation of Bax/BCl-2 ratio, activation of caspase-3/8/9, and downregulation of the Akt. Tumor Biol. 2014;35(3):1819-25.

5. Yen MH, Lee JJ, Yeh CF, Wang KC, Chiang YW, Chiang LC, Chang JS. Yakammaoto inhibited human coxsackievirus B4 (CVB4)-induced airway and renal tubular injuries by preventing viral attachment, internalization, and replication. J Ethnopharmacol. 2014;151(3):1056-63.

6. Vissiennon C, Nieber K, Kelber O, Butterweck V. Route of administration determines the anxiolytic activity of the flavonols kaempferol, quercetin and myricetin—are they prodrugs? J Nutr Biochem. 2012;23(7):733-40.

7. Kim DH, Jung EA, Sohng IS, Han JA, Kim TH, Han MJ. Intestinal bacterial metabolism of flavonoids and its relation to some biological activities. Arch Pharm Res. 1998;21:17-23.

8. Zhao HQ, Jiang ZH, Chang XM, Xue HT, Yahefu W, Zhang XY. 4-Hydroxyphenylacetic acid prevents acute APAP-induced liver injury by increasing phase ii and antioxidant enzymes in mice. Front Pharmacol. 2018;9:653.

9. Wen KC, Chang CS, Chien YC, Wang HW, Wu WC, Wu CS, Chiang HM. Tyrosol and its analogues inhibit alpha-melanocyte-stimulating hormone induced melanogenesis. Int J Mol Sci. 2013;14(12):23420-40.

10. Liu ZY, Xi RG, Zhang ZR, Li WP, Liu Y, Jin FG, Wang XB. 4-Hydroxyphenylacetic acid attenuated inflammation and edema via suppressing HIF-1 alpha in seawater aspiration-induced lung injury in rats. Int J Mol Sci. 2014;15(7):12861-84.

11. Mutsukado M, Yamada S. Methods for preparing 4-hydroxyphenylacetic acids. United States Patent 4412082; 1983.

12. Vallejos JC, Christidis Y. Preparation process for hydroxyphenylacetic acids. United States Patent US005395964A; 1995.

13. Averesch NJH, Kromer JO. Metabolic engineering of the shikimate pathway for production of aromatics and derived compounds-present and future strain construction strategies. Front Bioeng Biotechnol. 2018;6:32.

14. Wang J, Shen XL, Rey J, Yuan QP, Yan YJ. Recent advances in microbial production of aromatic natural products and their derivatives. Appl Microbiol Biotechnol. 2018;102(1):47-61.

15. Gupta A, Reizman IMB, Reisch CR, Prather KLJ. Dynamic regulation of metabolic flux in engineered bacteria using a pathway-independent quorum-sensing circuit. Nat Biotechnol. 2017;35(3):273-9.

16. Wei T, Cheng BY, Liu JZ. Genome engineering Escherichia coli for L-DOPA overproduction from glucose. Sci Rep-Uk. 2016;6:30080.

17. Datsenko KA, Wanner BL. One-step inactivation of chromosomal genes in Escherichia coli K-12 using PCR products. Proc Natl Acad Sci USA. 2000;97(12):6640-5.

18. Lee TS, Krupa RA, Zhang FZ, Hajimorad M, Holtz WJ, Prasad N, Lee SK, Keasling JD. BglBrick vectors and datasheets: a synthetic biology platform for gene expression. J Biol Eng. 2011;5(1):12.

19. Li XR, Tian GQ, Shen HJ, Liu JZ. Metabolic engineering of Escherichia coli to produce zeaxanthin. J Ind Microbiol Biotechnol. 2015;42(4):627-36.

20. Wang C, Yoon SH, Jang HJ, Chung YR, Kim JY, Choi ES, Kim SW. Metabolic engineering of Escherichia coli for alpha-farnesene production. Metab Eng. 2011;13(6):648-55.

21. Kirby J, Nishimoto M, Chow RWN, Baidoo EEK, Wang G, Martin J, Schackwitz W, Chan R, Fortman JL, Keasling JD. Enhancing terpene yield from sugars via novel routes to 1-deoxy-D-xylulose 5-phosphate. Appl Environ Microb. 2015;81(1):130-8.

22. Miyazaki K. MEGAWHOP cloning: a method of creating random mutagenesis libraries via megaprimer PCR of whole plasmids. Method Enzymol. 2011;498:399-406.

23. Pfleger BF, Pitera DJ, Smolke CD, Keasling JD. Combinatorial engineering of intergenic regions in operons tunes expression of multiple genes. Nat Biotechnol. 2006;24(8):1027-32. 
24. Li XL, Chen ZY, Wu YF, Yan YJ, Sun XX, Yuan QP. Establishing an artificial pathway for efficient biosynthesis of hydroxytyrosol. ACS Synth Biol. 2018;7(2):647-54

25. Liu X, Li XB, Jiang JL, Liu ZN, Qiao B, Li FF, Cheng JS, Sun XC, Yuan YJ, Qiao $\mathrm{JJ}$, et al. Convergent engineering of syntrophic Escherichia coli coculture for efficient production of glycosides. Metab Eng. 2018;47:243-53.

26. Chung D, Kim SY, Ahn JH. Production of three phenylethanoids, tyrosol, hydroxytyrosol, and salidroside, using plant genes expressing in Escherichia coli. Sci Rep-Uk. 2017:7:2578.

27. Satoh Y, Tajima K, Munekata M, Keasling JD, Lee TS. Engineering of a tyrosol-producing pathway, utilizing simple sugar and the central metabolic tyrosine, Escherichia coli. J Agric Food Chem. 2012;60(4):979-84.

28. Claus H, Decker H. Bacterial tyrosinases. Syst Appl Microbiol. 2006;29(1):3-14

29. Niu FX, He X, Wu YQ, Liu JZ. Enhancing production of pinene in Escherichia coli by using a combination of tolerance, evolution, and modular co-culture engineering. Front Microbiol. 2018;9:1623.

30. Pai A, Tanouchi Y, You LC. Optimality and robustness in quorum sensing (QS)-mediated regulation of a costly public good enzyme. Proc Natl Acad Sci USA. 2012;109(48):19810-5.
31. Soma Y, Hanai T. Self-induced metabolic state switching by a tunable cell density sensor for microbial isopropanol production. Metab Eng. 2015:30:7-15.

32. Kim EM, Woo HM, Tian T, Yilmaz S, Javidpour P, Keasling JD, Lee TS. Autonomous control of metabolic state by a quorum sensing (QS)-mediated regulator for bisabolene production in engineered E. coli. Metab Eng. 2017:44:325-36.

33. Williams TC, Averesch NJH, Winter G, Plan MR, Vickers CE, Nielsen LK, Kromer JO. Quorum-sensing linked RNA interference for dynamic metabolic pathway control in Saccharomyces cerevisiae. Metab Eng. 2015;29:124-34.

34. Shong J, Collins CH. Engineering the esaR promoter for tunable quorum sensing-dependent gene expression. ACS Synth Biol. 2013;2(10):568-75.

35. Shong J, Huang YM, Bystroff C, Collins CH. Directed evolution of the quorum-sensing regulator EsaR for increased signal sensitivity. ACS Chem Biol. 2013;8(4):789-95.
Ready to submit your research? Choose BMC and benefit from:

- fast, convenient online submission

- thorough peer review by experienced researchers in your field

- rapid publication on acceptance

- support for research data, including large and complex data types

- gold Open Access which fosters wider collaboration and increased citations

- maximum visibility for your research: over 100M website views per year

At BMC, research is always in progress.

Learn more biomedcentral.com/submissions 